PROCEEDINGS OF THE

AMERICAN MATHEMATICAL SOCIETY

Volume 136, Number 8, August 2008, Pages 2793-2802

S 0002-9939(08)09341-6

Article electronically published on March 27, 2008

\title{
A GRAPH-THEORETIC APPROACH TO THE METHOD OF GLOBAL LYAPUNOV FUNCTIONS
}

\author{
HONGBIN GUO, MICHAEL Y. LI, AND ZHISHENG SHUAI \\ (Communicated by Carmen C. Chicone)
}

\begin{abstract}
A class of global Lyapunov functions is revisited and used to resolve a long-standing open problem on the uniqueness and global stability of the endemic equilibrium of a class of multi-group models in mathematical epidemiology. We show how the group structure of the models, as manifested in the derivatives of the Lyapunov function, can be completely described using graph theory.
\end{abstract}

\section{INTRODUCTION}

Let a function $x \mapsto f(x) \in \mathbb{R}^{N}$ be defined in an open region $D \subset \mathbb{R}^{N}$ such that the differential equation

$$
x^{\prime}=f(x), \quad x \in D,
$$

has a unique solution $x\left(t, x_{0}\right)$ for each initial point $x_{0} \in D$. An equilibrium $\bar{x} \in D$ is globally stable in $D$ if it is locally stable and $x\left(t, x_{0}\right) \rightarrow \bar{x}$ as $t \rightarrow \infty$ for all $x_{0} \in D$. A function $x \mapsto V(x) \in \mathbb{R}^{1}$ is said to be a global Lyapunov function of (1.1) for $D$ if

$$
\dot{V}(x)=\operatorname{grad} V(x) \cdot f(x) \leq 0, \quad x \in D .
$$

A classical theorem of Lyapunov states that if (1) $V(x) \geq 0$ for $x \in D$ and $V(x)=0$ iff $x=\bar{x}$, and $(2) \dot{V}(x) \leq 0$ for $x \in D$ and $\dot{V}(x)=0$ iff $x=\bar{x}$, then $\bar{x}$ is globally stable in $D$. Lyapunov's theorem was further extended as the LaSalle Invariance Principle [12]: if $V(x)$ is a global Lyapunov function in $D$, then all omega limit sets of (1.1) are contained in the maximal compact invariant subset $K$ of $G=$ $\{x \in D: \dot{V}(x)=0\}$. In particular, if $D$ is positively invariant and $K=\{\bar{x}\}$, then $x\left(t, x_{0}\right) \rightarrow \bar{x}$ as $t \rightarrow \infty$. We note that this also implies the local stability of $\bar{x}$, since otherwise $K$ would contain a non-constant full orbit.

In the literature of ecological models, the region $D$ is typically in the positive cone of $\mathbb{R}^{N}$, and a class of Lyapunov functions

$$
V(x)=\sum_{k=1}^{N} a_{k}\left(x_{k}-\bar{x}_{k} \ln x_{k}\right)
$$

Received by the editors November 8, 2006.

2000 Mathematics Subject Classification. Primary 34D23, 92D30.

Key words and phrases. Lyapunov functions, multi-group epidemic models, global stability, graph theory.

(C)2008 American Mathematical Society Reverts to public domain 28 years from publication 
has proven useful; see e.g. 3, 9] and the references therein. Recently, this form of Lyapunov functions was applied to several single-group epidemic models and used to prove the global stability of a unique endemic equilibrium [4, 10]. In the present paper, to further demonstrate the applicability of this form of Lyapunov functions, we apply them to a class of $n$-group $(n \geq 2)$ epidemic models of SEIR type with bilinear incidence, described by the following system of equations:

$$
\left\{\begin{array}{l}
S_{k}^{\prime}=\Lambda_{k}-d_{k}^{S} S_{k}-\sum_{j=1}^{n} \beta_{k j} S_{k} I_{j}, \\
E_{k}^{\prime}=\sum_{j=1}^{n} \beta_{k j} S_{k} I_{j}-\left(d_{k}^{E}+\epsilon_{k}\right) E_{k}, \\
I_{k}^{\prime}=\epsilon_{k} E_{k}-\left(d_{k}^{I}+\gamma_{k}\right) I_{k} .
\end{array} \quad k=1,2, \cdots, n,\right.
$$

Here $S_{k}, E_{k}$, and $I_{k}$ denote the population in the $k$-th group that are susceptible to the disease, infected but non-infectious, and infectious, respectively. The parameters in the model are non-negative constants and summarized in the following list:

$\beta_{k j}:$ transmission coefficient between compartments $S_{k}$ and $I_{j}$, $d_{k}^{S}, d_{k}^{E}, d_{k}^{I} \quad: \quad$ natural death rates of $S, E, I$ compartments in the $k$-th group, respectively,

$\Lambda_{k} \quad$ : influx of individuals into the $k$-th group,

$\epsilon_{k}$ : rate of becoming infectious after latent period in the $k$-th group,

$\gamma_{k}:$ recovery rate of infectious individuals in the $k$-th group.

In particular, $\beta_{k j} \geq 0$, and $\beta_{k j}=0$ if there is no transmission of the disease between compartments $S_{k}$ and $I_{j}$. The matrix $B=\left(\beta_{k j}\right)$ encodes the patterns of contact and transmission among groups that are built into the model. Associated to $B$, one can construct a directed graph $L=G(B)$ whose vertex $k$ represents the $k$-th group, $k=1, \cdots, n$. A directed edge exists from vertex $k$ to vertex $j$ if and only if $\beta_{k j}>0$. Throughout the paper, we assume that $B$ is irreducible. This is equivalent to $G(B)$ being strongly connected (see Section 2). Biologically, this is the same as assuming that any two groups $k$ and $j$ have a direct or indirect route of transmission. More specifically, individuals in $I_{j}$ can infect ones in $S_{k}$ directly or indirectly. We also assume that $\epsilon_{k}>0$ and $d_{k}^{*}>0$, where $d_{k}^{*}=\min \left\{d_{k}^{S}, d_{k}^{E}, d_{k}^{I}+\gamma_{k}\right\}$. For more detailed discussions of the model and interpretations of parameters, we refer the reader to [16].

For each $k$, adding the three equations in (1.3) gives $\left(S_{k}+E_{k}+I_{k}\right)^{\prime} \leq \Lambda_{k}-$ $d_{k}^{*}\left(S_{k}+E_{k}+I_{k}\right)$. Hence $\lim \sup _{t \rightarrow \infty}\left(S_{k}+E_{k}+I_{k}\right) \leq \Lambda_{k} / d_{k}^{*}$. Similarly, from the $S_{k}$ equation we obtain $\lim \sup _{t \rightarrow \infty} S_{k} \leq \Lambda_{k} / d_{k}^{S}$. Therefore, omega limit sets of system (1.3) are contained in the following bounded region in the non-negative cone of $\mathbb{R}^{3 n}$ : $(1.4)$

$\Gamma=\left\{\left(S_{1}, E_{1}, I_{1}, \cdots, S_{n}, E_{n}, I_{n}\right) \in \mathbb{R}_{+}^{3 n} \quad \mid \quad S_{k} \leq \frac{\Lambda_{k}}{d_{k}^{S}}, S_{k}+E_{k}+I_{k} \leq \frac{\Lambda_{k}}{d_{k}^{*}}, 1 \leq k \leq n\right\}$.

It can be verified that region $\Gamma$ is positively invariant. System (1.3) always has the disease-free equilibrium $P_{0}=\left(S_{1}^{0}, 0,0, \cdots, S_{n}^{0}, 0,0\right)$ on the boundary of $\Gamma$, where $S_{k}^{0}=\Lambda_{k} / d_{k}^{S}$. An equilibrium $P^{*}=\left(S_{1}^{*}, E_{1}^{*}, I_{1}^{*}, \cdots, S_{n}^{*}, E_{n}^{*}, I_{n}^{*}\right)$ in the interior $\stackrel{\circ}{\Gamma}$ of $\Gamma$ is called an endemic equilibrium, where $S_{k}^{*}, E_{k}^{*}, I_{k}^{*}>0$ satisfy the equilibrium 
equations

$$
\begin{gathered}
\Lambda_{k}=d_{k}^{S} S_{k}^{*}+\sum_{j=1}^{n} \beta_{k j} S_{k}^{*} I_{j}^{*} \\
\left(d_{k}^{E}+\epsilon_{k}\right) E_{k}^{*}=\sum_{j=1}^{n} \beta_{k j} S_{k}^{*} I_{j}^{*}, \quad \epsilon_{k} E_{k}^{*}=\left(d_{k}^{I}+\gamma_{k}\right) I_{k}^{*}
\end{gathered}
$$

and

$$
\frac{\left(d_{k}^{E}+\epsilon_{k}\right)\left(d_{k}^{I}+\gamma_{k}\right)}{\epsilon_{k}} I_{k}^{*}=\sum_{j=1}^{n} \beta_{k j} S_{k}^{*} I_{j}^{*},
$$

which follows from (1.6).

Let

$$
R_{0}=\rho\left(M_{0}\right)
$$

denote the spectral radius of the matrix

$$
M_{0}=\left(\frac{\beta_{k j} \epsilon_{k} S_{k}^{0}}{\left(d_{k}^{E}+\epsilon_{k}\right)\left(d_{k}^{I}+\gamma_{k}\right)}\right)_{1 \leq k, j \leq n} .
$$

The parameter $R_{0}$ is referred to as the basic reproduction number. Its biological significance is that if $R_{0}<1$ the disease dies out while if $R_{0}>1$ the disease becomes endemic ([16, 17]). A long-standing open question in mathematical epidemiology is whether a multi-group model such as system (1.3) has a unique endemic equilibrium $P^{*}$ when $R_{0}>1$, and whether $P^{*}$ is globally stable when it is unique [16. We prove the following theorem, which settles this open problem for system (1.3), as well as other multi-group models that can be converted to the same form.

Theorem 1.1. Assume that $B=\left(\beta_{k j}\right)$ is irreducible. If $R_{0}>1$, then system (1.3) has a unique endemic equilibrium $P^{*}$, and $P^{*}$ is globally stable in $\stackrel{\circ}{\Gamma}$.

One of the earliest results on multi-group models is by Lajmanovich and Yorke [11 on a class of $n$-group SIS models for gonorrhea. The global stability of the unique endemic equilibrium is proved using a quadratic global Lyapunov function. Global stability results also exist for other types of multi-group models; see e.g., [1, 5, 6, 13, 15. The most recent result is Lin and So [13, for a class of SIRS models, in which the endemic equilibrium is shown to be globally stable if all $\beta_{k j}, k \neq j$, are small or if all $\gamma_{k}, 1 \leq k \leq n$, are small. Results in the opposite direction also exist in the literature. For a class of $n$-group SIR models with proportionate incidence, uniqueness of endemic equilibria may not hold when $R_{0}>1$ [7, 16].

Our proof of Theorem 1.1 uses the form of global Lyapunov functions given in (1.2). Compared to results in [4, 10, the group structure in system (1.3) greatly increases the complexity exhibited in the derivatives of the Lyapunov function $V$. The key to our analysis is a complete description of the patterns exhibited in the derivative $\dot{V}$ using graph theory. As structured models are used to describe more complicated biological problems, we expect that this class of Lyapunov functions, together with our graph theoretical analysis, will have much wider applicability. 


\section{Preliminaries}

A non-negative matrix $E$ is reducible if, for some permutation matrix $Q$,

$$
Q E Q^{T}=\left[\begin{array}{cc}
E_{1} & 0 \\
E_{2} & E_{3}
\end{array}\right]
$$

and $E_{1}, E_{3}$ are square matrices. Otherwise, $E$ is irreducible. Irreducibility of $E$ can be checked using the associated directed graphs. The directed graph $G(E)$ associated with $E=\left(e_{k j}\right)$ has vertices $\{1,2, \cdots, n\}$ with a directed arc $(k, j)$ from $k$ to $j$ iff $e_{k j} \neq 0$. It is strongly connected if any two distinct vertices are joined by an oriented path. Matrix $E$ is irreducible if and only if $G(E)$ is strongly connected.

A tree is a connected graph with no cycles. A subtree $T$ of a graph $G$ is said to be spanning if $T$ contains all the vertices of $G$. A directed tree is a tree in which each edge has been replaced by an arc directed one way or the other. A directed tree is said to be rooted at a vertex, called the root, if every arc is oriented in the direction towards the root. An oriented cycle in a directed graph is a simple closed oriented path. A unicyclic graph is a directed graph consisting of a collection of disjoint rooted directed trees whose roots are on an oriented cycle. We refer the reader to $[8,14$ for more details.

When a directed arc from the root to any non-root vertex is added to a directed rooted tree, we obtain a unicyclic graph. See Figure 1. When we perform this operation in all possible ways to all possible directed rooted trees on a given set of vertices, we obtain all possible unicyclic graphs on these vertices with each unicyclic graph counted separately for each cyclic arc it contains. This observation will play a crucial role in the proof of Theorem 1.1.

Now consider the linear system

$$
\bar{B} v=0
$$

where

$$
\bar{B}=\left[\begin{array}{cccc}
\sum_{l \neq 1} \bar{\beta}_{1 l} & -\bar{\beta}_{21} & \cdots & -\bar{\beta}_{n 1} \\
-\bar{\beta}_{12} & \sum_{l \neq 2} \bar{\beta}_{2 l} & \cdots & -\bar{\beta}_{n 2} \\
\vdots & \vdots & \ddots & \vdots \\
-\bar{\beta}_{1 n} & -\bar{\beta}_{2 n} & \cdots & \sum_{l \neq n} \bar{\beta}_{n l}
\end{array}\right],
$$

and $\bar{\beta}_{k j} \geq 0,1 \leq k, j \leq n$. Let $L=G(B)$ denote the directed graph associated with matrix $B$ (and $\left.\left(\bar{\beta}_{k j}\right)\right)$, and $C_{j k}$ denote the cofactor of the $(j, k)$ entry of $\bar{B}$.

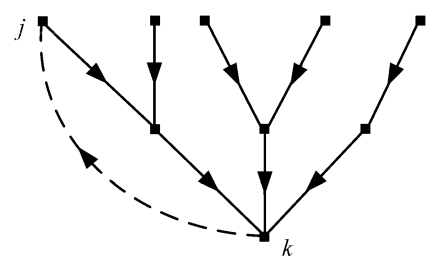

Figure 1. A unicyclic graph formed by adding a directed arc $(k, j)$ to a directed tree rooted at $k$. 
Lemma 2.1. Assume $\left(\bar{\beta}_{k j}\right)_{n \times n}$ is irreducible and $n \geq 2$. Then the following results hold:

(1) The solution space of system (2.1) has dimension 1 , with a basis $\left(v_{1}, v_{2}, \cdots\right.$, $\left.v_{n}\right)=\left(C_{11}, C_{22}, \cdots, C_{n n}\right)$.

(2) For $1 \leq k \leq n$,

$$
C_{k k}=\sum_{T \in \mathbb{T}_{k}} \prod_{(r, m) \in E(T)} \bar{\beta}_{r m}>0,
$$

where $\mathbb{T}_{k}$ is the set of all directed spanning subtrees of $L$ that are rooted at vertex $k$, and $E(T)$ denotes the set of directed arcs in a directed tree $T$.

Proof. Since the sum of each column in $\bar{B}$ equals zero, we have $C_{j k}=C_{l k}, 1 \leq$ $j, k, l \leq n$. From $\operatorname{det}(\bar{B})=0$, we know that $\left(C_{11}, C_{12}, \cdots, C_{1 n}\right)$, and thus $\left(C_{11}, C_{22}\right.$, $\left.\cdots, C_{n n}\right)$, is a solution of (2.1). By the Matrix-Tree Theorem ([14, Theorem 5.5] or [8, page 378]), the expansion of each $(n-1)$ principal minor of $\bar{B}$ can be expressed as

$$
C_{k k}=\sum_{T \in \mathbb{T}_{k}} \prod_{(r, m) \in E(T)} \bar{\beta}_{r m} .
$$

Since $\left(\bar{\beta}_{k j}\right)$ is irreducible, its associated directed graph $L$ is strongly connected. For each $k$, at least one term in $\sum_{T \in \mathbb{T}_{k}} \prod_{(r, m) \in E(T)} \bar{\beta}_{r m}$ is positive, and thus $C_{k k}>0$. This implies $\operatorname{rank}(\bar{B})=n-1$, and the solution space of (2.1) has dimension 1 .

As an illustration of (2.3), let $n=3$ and $\mathbb{T}_{1}=\left\{T_{1}^{1}, T_{1}^{2}, T_{1}^{3}\right\}$ be the set of all directed trees with vertices $\{1,2,3\}$ rooted at vertex 1 . See Figure 2 . Then, $E\left(T_{1}^{1}\right)=$ $\{(3,2),(2,1)\}, E\left(T_{1}^{2}\right)=\{(2,1),(3,1)\}, E\left(T_{1}^{3}\right)=\{(2,3),(3,1)\}$. Therefore,

$$
\begin{aligned}
C_{11} & =\operatorname{det}\left|\begin{array}{cc}
\bar{\beta}_{21}+\bar{\beta}_{23} & -\bar{\beta}_{32} \\
-\bar{\beta}_{23} & \bar{\beta}_{31}+\bar{\beta}_{32}
\end{array}\right| \\
& =\bar{\beta}_{32} \bar{\beta}_{21}+\bar{\beta}_{21} \bar{\beta}_{31}+\bar{\beta}_{23} \bar{\beta}_{31} \\
& =\sum_{T_{1}^{i} \in \mathbb{T}_{1}} \prod_{(r, m) \in E\left(T_{1}^{i}\right)} \bar{\beta}_{r m} .
\end{aligned}
$$

Let $R_{0}$ be defined in (1.8). The following result for system (1.3) is known in the literature, at least for some special classes of system (1.3), and its proof is standard (see [6, 15, 17]).

Proposition 2.2. Assume $B=\left(\beta_{k j}\right)$ is irreducible. Then the following statements hold:
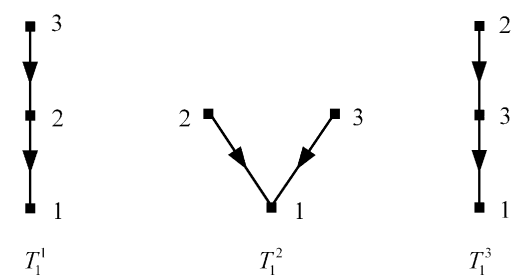

Figure 2. Three-vertex directed trees that are rooted at 1. 
(1) If $R_{0} \leq 1$, then $P_{0}$ is the unique equilibrium and it is globally stable in $\Gamma$.

(2) If $R_{0}>1$, then $P_{0}$ is unstable and system (1.3) is uniformly persistent in $\stackrel{\circ}{\Gamma}$.

Uniform persistence of (1.3), together with uniform boundedness of solutions in $\stackrel{\circ}{\Gamma}$, implies the existence of an equilibrium of $(1.3)$ in $\stackrel{\circ}{\Gamma}$ ([2, Theorem 2.8.6]).

Corollary 2.3. Assume $B=\left(\beta_{k j}\right)$ is irreducible. If $R_{0}>1$, then (1.3) has at least one endemic equilibrium.

\section{Proof of Theorem 1.1}

Let $P^{*}=\left(S_{1}^{*}, E_{1}^{*}, I_{1}^{*}, \cdots, S_{n}^{*}, E_{n}^{*}, I_{n}^{*}\right), S_{k}^{*}, E_{k}^{*}, I_{k}^{*}>0$ for $1 \leq k \leq n$, denote an endemic equilibrium whose existence is established in Corollary 2.3. We prove that $P^{*}$ is globally stable when $R_{0}>1$. In particular, this implies that the endemic equilibrium is unique in $\stackrel{\circ}{\Gamma}$ when it exists. Choose

$$
\bar{\beta}_{k j}=\beta_{k j} S_{k}^{*} I_{j}^{*}, \quad 1 \leq k, j \leq n, \quad n \geq 2,
$$

and matrix $\bar{B}$ as given in (2.2). Since $B=\left(\beta_{k j}\right)$ is irreducible, we know matrix $\left(\bar{\beta}_{k j}\right)$ is irreducible. Let $\left\{v_{1}, \cdots, v_{n}\right\}, v_{k}>0$, be a basis for system (2.1), $\bar{B} v=0$, as described in Lemma 2.1. Set

$$
V=\sum_{k=1}^{n} v_{k}\left[\left(S_{k}-S_{k}^{*} \ln S_{k}\right)+\left(E_{k}-E_{k}^{*} \ln E_{k}\right)+\frac{d_{k}^{E}+\epsilon_{k}}{\epsilon_{k}}\left(I_{k}-I_{k}^{*} \ln I_{k}\right)\right] .
$$

Differentiating $V$ along solutions to (1.3) and using (1.5), (1.6), (1.7), we obtain

$$
\begin{aligned}
\dot{V}= & \sum_{k=1}^{n} v_{k}\left[\Lambda_{k}-d_{k}^{S} S_{k}-\sum_{j=1}^{n} \beta_{k j} S_{k} I_{j}-\Lambda_{k} \frac{S_{k}^{*}}{S_{k}}+d_{k}^{S} S_{k}^{*}+\sum_{j=1}^{n} \beta_{k j} S_{k}^{*} I_{j}\right. \\
& +\sum_{j=1}^{n} \beta_{k j} S_{k} I_{j}-\left(d_{k}^{E}+\epsilon_{k}\right) E_{k}-\sum_{j=1}^{n} \beta_{k j} \frac{E_{k}^{*} S_{k} I_{j}}{E_{k}}+\left(d_{k}^{E}+\epsilon_{k}\right) E_{k}^{*} \\
& +\left(d_{k}^{E}+\epsilon_{k}\right) E_{k}-\frac{\left(d_{k}^{E}+\epsilon_{k}\right)\left(d_{k}^{I}+\gamma_{k}\right)}{\epsilon_{k}} I_{k}-\left(d_{k}^{E}+\epsilon_{k}\right) \frac{I_{k}^{*} E_{k}}{I_{k}} \\
& \left.+\frac{\left(d_{k}^{E}+\epsilon_{k}\right)\left(d_{k}^{I}+\gamma_{k}\right)}{\epsilon_{k}} I_{k}^{*}\right] \\
= & \sum_{k=1}^{n} v_{k}\left[d_{k}^{S} S_{k}^{*}\left(2-\frac{S_{k}^{*}}{S_{k}}-\frac{S_{k}}{S_{k}^{*}}\right)+\left(\sum_{j=1}^{n} \beta_{k j} S_{k}^{*} I_{j}-\frac{\left(d_{k}^{E}+\epsilon_{k}\right)\left(d_{k}^{I}+\gamma_{k}\right)}{\epsilon_{k}} I_{k}\right)\right. \\
& \left.+\left(3 \sum_{j=1}^{n} \beta_{k j} S_{k}^{*} I_{j}^{*}-\sum_{j=1}^{n} \beta_{k j} I_{j}^{*} \frac{\left(S_{k}^{*}\right)^{2}}{S_{k}}-\sum_{j=1}^{n} \beta_{k j} S_{k} I_{j} \frac{E_{k}^{*}}{E_{k}}-\left(d_{k}^{E}+\epsilon_{k}\right) E_{k} \frac{I_{k}^{*}}{I_{k}}\right)\right] \\
\leq & \sum_{k=1}^{n} v_{k}\left[\left(\sum_{j=1}^{n} \beta_{k j} S_{k}^{*} I_{j}-\frac{\left(d_{k}^{E}+\epsilon_{k}\right)\left(d_{k}^{I}+\gamma_{k}\right)}{\epsilon_{k}} I_{k}\right)\right. \\
& \left.+\left(3 \sum_{j=1}^{n} \beta_{k j} S_{k}^{*} I_{j}^{*}-\sum_{j=1}^{n} \beta_{k j} I_{j}^{*} \frac{\left(S_{k}^{*}\right)^{2}}{S_{k}}-\sum_{j=1}^{n} \beta_{k j} S_{k} I_{j} \frac{E_{k}^{*}}{E_{k}}-\left(d_{k}^{E}+\epsilon_{k}\right) E_{k} \frac{I_{k}^{*}}{I_{k}}\right)\right]
\end{aligned}
$$


since $S_{k}^{*} / S_{k}+S_{k} / S_{k}^{*} \geq 2$, with equality holding if and only if $S_{k}=S_{k}^{*}$.

To simplify this we observe that since $\bar{B} v=0$, it follows readily that

$$
\sum_{j=1}^{n} \bar{\beta}_{j k} v_{j}=\sum_{i=1}^{n} \bar{\beta}_{k i} v_{k}
$$

or, appealing to (3.1) and (1.7), that

$$
\sum_{j=1}^{n} \beta_{j k} S_{j}^{*} I_{k}^{*} v_{j}=\sum_{i=1}^{n} \beta_{k i} S_{k}^{*} I_{i}^{*} v_{k}=\frac{\left(d_{k}^{E}+\epsilon_{k}\right)\left(d_{k}^{I}+\gamma_{k}\right)}{\epsilon_{k}} I_{k}^{*} v_{k}
$$

for $1 \leq k \leq n$. This implies that

$$
\sum_{k=1}^{n} v_{k} \sum_{j=1}^{n} \beta_{k j} S_{k}^{*} I_{j}=\sum_{k=1}^{n} I_{k} \sum_{j=1}^{n} \beta_{j k} S_{j}^{*} v_{j}=\sum_{k=1}^{n} \frac{\left(d_{k}^{E}+\epsilon_{k}\right)\left(d_{k}^{I}+\gamma_{k}\right)}{\epsilon_{k}} I_{k} v_{k} .
$$

Consequently, the contributions from the first two double sums in the right-handside of (3.3) cancel, so it follows from (3.3), appealing again to relation (3.1), that

$$
\begin{aligned}
\stackrel{\bullet}{V} & \leq \sum_{k=1}^{n} v_{k}\left(3 \sum_{j=1}^{n} \bar{\beta}_{k j}-\sum_{j=1}^{n} \bar{\beta}_{k j} \frac{S_{k}^{*}}{S_{k}}-\sum_{j=1}^{n} \bar{\beta}_{k j} \frac{S_{k} I_{j} E_{k}^{*}}{S_{k}^{*} I_{j}^{*} E_{k}}-\left(d_{k}^{E}+\epsilon_{k}\right) E_{k} \frac{I_{k}^{*}}{I_{k}}\right) \\
& =\sum_{k, j=1}^{n} v_{k} \bar{\beta}_{k j}\left(3-\frac{S_{k}^{*}}{S_{k}}-\frac{S_{k} I_{j} E_{k}^{*}}{S_{k}^{*} I_{j}^{*} E_{k}}-\frac{E_{k} I_{k}^{*}}{E_{k}^{*} I_{k}}\right)=: H_{n}\left(S_{1}, E_{1}, I_{1}, \cdots, S_{n}, E_{n}, I_{n}\right) .
\end{aligned}
$$

In the following, we show $H_{n} \leq 0$ for all $\left(S_{1}, E_{1}, I_{1}, \cdots, S_{n}, E_{n}, I_{n}\right) \in \stackrel{\circ}{\Gamma}$.

The products of factors $\bar{\beta}_{r m}$ in the expression for $v_{k}$ given in (2.3) can be interpreted as weights of directed spanning subtrees $T$ of $L$ that are rooted at vertex $k$. Consequently, each product in $v_{k} \bar{\beta}_{k j}$ can be interpreted as the weight of a unicyclic graph $Q$ obtained from such a tree $T$ by adding an $\operatorname{arc}(k, j)$ directed from the root vertex $k$ to vertex $j$; see Figure 1 . Such a product can be written as

$$
\prod_{(r, m) \in E(Q)} \bar{\beta}_{r m}
$$

where the product is over the $\operatorname{arcs}(r, m)$ in $Q$, and the same product is obtained for each $\operatorname{arc}(k, j)$ in the unique cycle $C Q$ of $Q$. It is not difficult to see that the double sum in $H_{n}$ can be interpreted as a sum over all the arcs in the cycles of all the unicyclic subgraphs $Q$ of $L$. Hence $H_{n}$ can be rewritten as

$$
H_{n}=\sum_{Q} H_{n, Q}
$$

where

$$
\begin{aligned}
H_{n, Q} & =\prod_{(r, m) \in E(Q)} \bar{\beta}_{r m} \cdot \sum_{(k, j) \in E(C Q)}\left(3-\frac{S_{k}^{*}}{S_{k}}-\frac{S_{k} I_{j} E_{k}^{*}}{S_{k}^{*} I_{j}^{*} E_{k}}-\frac{E_{k} I_{k}^{*}}{E_{k}^{*} I_{k}}\right) \\
& =\prod_{(r, m) \in E(Q)} \bar{\beta}_{r m} \cdot\left(3 l-\sum_{(k, j) \in E(C Q)}\left(\frac{S_{k}^{*}}{S_{k}}-\frac{S_{k} I_{j} E_{k}^{*}}{S_{k}^{*} I_{j}^{*} E_{k}}-\frac{E_{k} I_{k}^{*}}{E_{k}^{*} I_{k}}\right)\right),
\end{aligned}
$$

and where $l=l(Q)$ denotes the number of $\operatorname{arcs}$ in $C Q$. 
It is not difficult to see that

$$
\prod_{(k, j) \in E(C Q)} \frac{S_{k}^{*}}{S_{k}} \cdot \frac{S_{k} I_{j} E_{k}^{*}}{S_{k}^{*} I_{j}^{*} E_{k}} \cdot \frac{E_{k} I_{k}^{*}}{E_{k}^{*} I_{k}}=\prod_{(k, j) \in E(C Q)} \frac{I_{j} I_{k}^{*}}{I_{j}^{*} I_{k}}=1
$$

for each unicyclic graph $Q$. Therefore,

$$
\sum_{(k, j) \in E(C Q)}\left(\frac{S_{k}^{*}}{S_{k}}+\frac{S_{k} I_{j} E_{k}^{*}}{S_{k}^{*} I_{j}^{*} E_{k}}+\frac{E_{k} I_{k}^{*}}{E_{k}^{*} I_{k}}\right) \geq 3 l,
$$

and thus $H_{n, Q} \leq 0$ for each $Q$, and

$$
H_{n, Q}=0 \Longleftrightarrow \frac{S_{k}^{*}}{S_{k}}=\frac{S_{k} I_{j} E_{k}^{*}}{S_{k}^{*} I_{j}^{*} E_{k}}=\frac{E_{k} I_{k}^{*}}{E_{k}^{*} I_{k}}, \quad(k, j) \in E(C Q) .
$$

Therefore,

$$
H_{n}=\sum_{Q} H_{n, Q} \leq 0
$$

We have shown that $\dot{V} \leq 0$ for all $\left(S_{1}, E_{1}, I_{1}, \cdots, S_{n}, E_{n}, I_{n}\right) \in \stackrel{\circ}{\Gamma}$, and that $\dot{V}=0$ iff $S_{k}=S_{k}^{*}$ and $H_{n}=0$. We claim that if $S_{k}=S_{k}^{*}, 1 \leq k \leq n$, then

$$
H_{n}=0 \Longleftrightarrow E_{k}=a E_{k}^{*}, \quad I_{k}=a I_{k}^{*}, \quad k=1,2, \cdots, n,
$$

where $a$ is an arbitrary positive number. We first note that it follows from (3.5) that

$$
\frac{I_{k}}{I_{k}^{*}}=\frac{E_{k}}{E_{k}^{*}}=\frac{I_{j}}{I_{j}^{*}}
$$

for every arc $(k, j)$ that belongs to the cycle of some unicyclic subgraph $Q$ of $L$. But $L$ is strongly connected, so, clearly, every arc $(k, j)$ of $L$ belongs to the cycle of at least one such subgraph $Q$ and, hence, (3.7) holds for every arc $(k, j)$. Now let $p$ and $q$ denote any two distinct vertices of the graph. Then, by strong connectivity, there exists an oriented path from $p$ to $q$. If we apply (3.7) to the arcs of this path consecutively, we find that $\frac{I_{p}}{I_{p}^{*}}=\frac{I_{q}}{I_{q}^{*}}$; then it follows from (3.7) that $\frac{E_{p}}{E_{p}^{*}}=\frac{I_{p}}{I_{p}^{*}}=\frac{I_{q}}{I_{q}^{*}}=$ $\frac{E_{q}}{E_{q}^{*}}$ as well. Since $p$ and $q$ are arbitrary, this suffices to prove (3.6).

From (3.3) and (3.6), we know that $\dot{V}=0$ iff $S_{k}=S_{k}^{*}, E_{k}=a E_{k}^{*}, I_{k}=a I_{k}^{*}, k=$ $1,2, \cdots, n$. Substituting $S_{k}=S_{k}^{*}, E_{k}=a E_{k}^{*}$, and $I_{k}=a I_{k}^{*}$ into the first equation of system (1.3), we obtain

$$
0=\Lambda_{k}-d_{k}^{S} S_{k}^{*}-a \sum_{j=1}^{n} \beta_{k j} S_{k}^{*} I_{j}^{*}
$$

Since the right-hand-side of (3.8) is strictly decreasing in $a$, we know, by (1.5), that (3.8) holds iff $a=1$, namely at $P^{*}$. Therefore, the only compact invariant subset of the set where $\dot{V}=0$ is the singleton $\left\{P^{*}\right\}$. By the LaSalle Invariance Principle, $P^{*}$ is globally stable in $\stackrel{\circ}{\Gamma}$ if $R_{0}>1$. This completes the proof of Theorem 1.1. 


\section{ACKNOWLEDGMENTS}

This research was supported in part by grants from the Natural Science and Engineering Research Council of Canada (NSERC) and the Canada Foundation for Innovation (CFI). The first author acknowledges the support of a PIMS Graduate Scholarship at the University of Alberta. The third author acknowledges the support of an Izaak Walton Killam Memorial Scholarship. The authors would like to thank an anonymous referee whose suggestions have greatly improved the exposition of the manuscript.

\section{REFERENCES}

[1] E. Beretta and V. Capasso, Global stability results for a multigroup SIR epidemic model, in: T. G. Hallam, L. J. Gross, and S. A. Levin (Eds.), Mathematical Ecology, World Scientific, Teaneck, NJ, 1988, pp. 317-342. MR1040340 (91c:92074)

[2] N. P. Bhatia and G. P. Szegö, Dynamical Systems: Stability Theory and Applications, Lecture Notes in Mathematics, Vol. 35, Springer, Berlin, 1967. MR.0219843 (36:2917)

[3] H. I. Freedman and J. W. -H. So, Global stability and persistence of simple food chains, Math. Biosci. 76 (1985) 69-86. MR809991 (87g:92040)

[4] H. Guo and M. Y. Li, Global dynamics of a staged progression model for infectious diseases, Math. Biosci. Eng. 3 (2006) 513-525. MR2217225 (2006m:92086)

[5] H. W. Hethcote, An immunization model for a heterogeneous population, Theor. Popu. Biol. 14 (1978) 338-349. MR520143 (80k:92009)

[6] H. W. Hethcote and H. R. Thieme, Stability of the endemic equilibrium in epidemic models with subpopulations, Math. Biosci. 75 (1985) 205-227. MR810991 (87c:92046)

[7] W. Huang, K. L. Cooke, and C. Castillo-Chavez, Stability and bifurcation for a multiplegroup model for the dynamics of HIV/AIDS transmission, SIAM J. Appl. Math. 52 (1992) 835-854. MR.1163809 (93d:92009)

[8] D. E. Knuth, The Art of Computer Programming, Vol. 1, 3rd ed., Addison-Wesley, Reading, MA, 1997. MR0286317 (44:3530)

[9] A. Korobeinikov, A Lyapunov function for Leslie-Gower predator-prey models, Appl. Math. Lett. 14 (2001) 697-699. MR.1836072

[10] A. Korobeinikov and P.K. Maini, A Lyapunov function and global properties for SIR and SEIR epidemiological models with nonlinear incidence, Math. Biosci. Eng. 1 (2004) 57-60. MR:2130156 (2005k:92065)

[11] A. Lajmanovich and J. A. York, A deterministic model for gonorrhea in a nonhomogeneous population, Math. Biosci. 28 (1976) 221-236. MR0403726 (53:7537)

[12] J. P. LaSalle, The Stability of Dynamical Systems, Regional Conference Series in Applied Mathematics, SIAM, Philadelphia, PA, 1976. MR0481301 (58:1426)

[13] X. Lin and J. W.-H. So, Global stability of the endemic equilibrium and uniform persistence in epidemic models with subpopulations, J. Austral. Math. Soc. Ser. B 34 (1993) 282-295. MR:1197481 (93k:92010)

[14] J. W. Moon, Counting Labelled Trees, Canadian Mathematical Congress, Montreal, 1970. MR0274333 (43:98)

[15] H. R. Thieme, Local stability in epidemic models for heterogeneous populations, in: V. Capasso, E. Grosso, and S.L. Paveri-Fontana (Eds.), Mathematics in Biology and Medicine, Lecture Notes in Biomathematics, Vol. 57, Springer, Berlin, 1985, pp. 185-189. MR812889

[16] H. R. Thieme, Mathematics in Population Biology, Princeton University Press, Princeton, NJ, 2003. MR1993355 (2004m:92030)

[17] P. van den Driessche and J. Watmough, Reproduction numbers and sub-threshold endemic equilibria for compartmental models of disease transmission, Math. Biosci. 180 (2002) 29-48. MR.1950747 (2003m:92071) 
Department of Mathematical and Statistical Sciences, University of Alberta, Edmonton, Alberta, T6G 2G1, Canada

Current address: Department of Mathematics and Statistics, York University, Toronto, Ontario, M3J 1P3, Canada

E-mail address: hguo@math.ualberta.ca

Department of Mathematical and Statistical Sciences, University of Alberta, Edmonton, Alberta, T6G 2G1, Canada

E-mail address: mli@math.ualberta.ca

Department of Mathematical and Statistical Sciences, University of Alberta, Edmonton, Alberta, T6G 2G1, Canada

E-mail address: zshuai@math.ualberta.ca 